\title{
Preprint of:
}

T. A. Nieminen, H. Rubinsztein-Dunlop, N. R. Heckenberg and A. I. Bishop

"Numerical Modelling of Optical Trapping"

Computer Physics Communications 142, 468-471 (2001)

\section{Numerical Modelling of Optical Trapping}

\author{
T. A. Nieminen, H. Rubinsztein-Dunlop, N. R. Heckenberg and A. I. Bishop ${ }^{a}$ \\ ${ }^{a}$ Centre for Laser Science, Department of Physics, \\ The University of Queensland, Brisbane QLD 4072, Australia
}

Optical trapping is a widely used technique, with many important applications in biology and metrology. Complete modelling of trapping requires calculation of optical forces, primarily a scattering problem, and nonoptical forces. The T-matrix method is used to calculate forces acting on spheroidal and cylindrical particles.

PACS codes: 42.50.Vk Mechanical effects of light; 42.25.Fx Diffraction and scattering

Keywords: trapping, optical tweezers, radiation pressure

\section{Introduction}

Optical trapping provide three-dimensional confinement and manipulation of microscopic particles by a focussed laser beam. Optical trapping is a powerful and widespread technique, with the single-beam gradient trap (also known as optical tweezers) in use for a large number of biological and other applications.

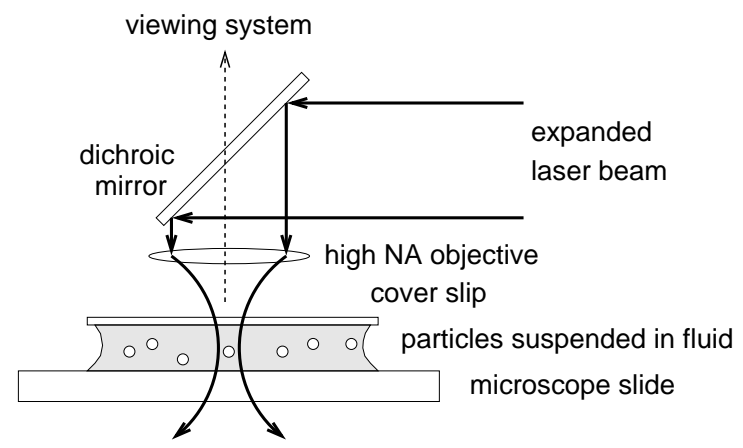

Figure 1. Schematic diagram of a typical optical tweezers setup

The trapping beam applies optical forces (usually divided into a gradient force, acting towards areas of higher irradiance, and scattering forces, including absorption and reflection forces) to the particle.

The optical forces and torques result from the transfer of momentum and angular momentum from the trapping beam to the particle. Various approximate methods such as geometric optics or Rayleigh approximations are often used for the calculation of the optical forces. Such approximate methods are not necessary, since electromagnetic scattering theory can be used for the calculation of forces, avoiding the limited ranges of applicability of the approximate methods.

Other forces will also affect the motion of the particle. The most important of these forces, gravity, bouyancy, and viscous drag as the particle moves through the surrounding fluid, are readily taken into account.

\section{Trapping as a scattering problem}

The optical forces and torques applied to the particle result from the transfer of momentum and angular momentum from the trapping beam to the particle. The total momentum transfer can be found by solving the electromagnetic scattering problem. A variety of numerical methods can be used - finite element method, FDTD, discrete dipole approximation [1], the T-matrix method [23], etc.

A number of these have been used for optical force calculations, including forces in optical traps 45. One method, however, stands out 
as ideal for trapping force calculations - the Tmatrix method. The T-matrix method can be considered an extension of Mie theory to arbitrarily shaped particles with arbitrary illumination. The main advantage of the T-matrix method is that trapping calculations usually involve repeated calculation of the scattering for the same particle under differing illumination. In this case, the T-matrix need only be calculated once, since it is independent of the fields, whereas methods such as FEM, FDTD and DDA will require the entire calculation to be repeated.

In the T-matrix method, the incident trapping field illuminating the particle is expressed as a sum of regular vector spherical wave functions (VSWFs):

$$
\begin{array}{r}
\mathbf{E}_{\mathrm{inc}}(\mathbf{r})=\sum_{n=1}^{\infty} \sum_{m=-n}^{n}\left[a_{m n} \mathbf{R} \mathbf{g} \mathbf{M}_{m n}(k \mathbf{r})+\right. \\
\left.b_{m n} \mathbf{R g} \mathbf{N}_{m n}(k \mathbf{r})\right]
\end{array}
$$

where

$$
\begin{gathered}
\mathbf{R g M}_{m n}(k \mathbf{r})=(-1)^{m} d_{n} \exp (i m \phi) \times \\
j_{n}(k r) \mathbf{C}_{m n}(\theta), \\
\mathbf{R g}_{m n}(k \mathbf{r})=(-1)^{m} d_{n} \exp (i m \phi) \times \\
\left\{\frac{n(n+1)}{k r} j_{n}(k r) \mathbf{P}_{m n}(\theta)+\right. \\
\left.\left[j_{n-1}(k r)-\frac{n}{k r} j_{n}(k r)\right] \mathbf{B}_{m n}(\theta)\right\}, \\
\mathbf{B}_{m n}(\theta)=\hat{\theta} \frac{d}{d \theta} d_{0 m}^{n}(\theta)+\hat{\phi} \frac{i m}{\sin \theta} d_{0 m}^{n}(\theta), \\
\mathbf{C}_{m n}(\theta)=\hat{\theta} \frac{i m}{\sin \theta} d_{0 m}^{n}(\theta)-\hat{\phi} \frac{d}{d \theta} d_{0 m}^{n}(\theta), \\
\mathbf{P}_{m n}(\theta)=\hat{r} d_{0 m}^{n}(\theta), \\
d_{n}=\left(\frac{2 n+1}{4 \pi n(n+1)}\right)^{\frac{1}{2}},
\end{gathered}
$$

$j_{n}(k r)$ are spherical Bessel functions, and $d_{0 m}^{n}(\theta)$ are Wigner $d$ functions.

Similarly, the scattered fields are expressed as a VSWF expansion. In this case, since the far field must be an outgoing radiation field,

$$
\begin{array}{r}
\mathbf{E}_{\text {scat }}(\mathbf{r})=\sum_{n=1}^{\infty} \sum_{m=-n}^{n}\left[p_{m n} \mathbf{M}_{m n}(k \mathbf{r})\right. \\
\left.+q_{m n} \mathbf{N}_{m n}(k \mathbf{r})\right]
\end{array}
$$

where $\mathbf{M}_{m n}(k \mathbf{r})$ and $\mathbf{N}_{m n}(k \mathbf{r})$ are the same as $\mathbf{R g M}_{m n}(k \mathbf{r})$ and $\mathbf{R g} \mathbf{N}_{m n}(k \mathbf{r})$, with the spherical Bessel functions replaced by spherical Hankel functions of the first kind, $h_{n}^{(1)}(k r)$.

From the linearity of the Maxwell equations, there is a linear relationship between the incident and scattered fields:

$p_{m n}=\sum_{m^{\prime} n^{\prime}} T_{m n m^{\prime} n^{\prime}}^{(11)} a_{m^{\prime} n^{\prime}}+T_{m n m^{\prime} n^{\prime}}^{(12)} b_{m^{\prime} n^{\prime}}$
$q_{m n}=\sum_{m^{\prime} n^{\prime}} T_{m n m^{\prime} n^{\prime}}^{(21)} a_{m^{\prime} n^{\prime}}+T_{m n m^{\prime} n^{\prime}}^{(22)} b_{m^{\prime} n^{\prime}}$

The T-matrix can be calculated using the extended boundary condition method (EBCM) 2 3]. For spherical particles, the T-matrix becomes diagonal, and the non-zero elements are the usual Mie coefficients. For rotationally symmetric particles, the T-matrix is diagonal with respect to $n$. Computer codes to calculate T-matrices for such rotationally symmetric particles are available 6 .

\section{Representation of the trapping beam}

The use of the T-matrix method for scattering calculations requires that the trapping beam be represented in terms of vector spherical wave functions, that is, the coefficients $a_{m n}$ and $b_{m n}$ in equation (11) need to be found. The regular VSWFs $\mathbf{R g} \mathbf{M}_{m n}$ and $\mathbf{R g} \mathbf{N}_{m n}$ provide a complete set of modes or partial waves, each individually satisfying the Maxwell equations, which can be used to represent any incident electromagnetic wave. For the simple case of a plane wave, $\mathbf{E}(\mathbf{r})=\mathbf{E}_{0} \exp (i \mathbf{k} \cdot \mathbf{r})$, with $\mathbf{k}$ in the direction $(\theta, \phi)$, the expansion coefficients are 23 ]

$a_{m n}=4 \pi(-1)^{m} i^{n} d_{n} \mathbf{C}_{m n}^{\star} \cdot \mathbf{E}_{0} \exp (-i m \phi)$
$b_{m n}=4 \pi(-1)^{m} i^{n-1} d_{n} \mathbf{B}_{m n}^{\star} \cdot \mathbf{E}_{0} \exp (-i m \phi)$.

Note that the amplitude vector $\mathbf{E}_{0}$ contains the information regarding the polarisation and phase of the wave, and can be complex.

In an optical trap, the incident field is usually a strongly focussed Gaussian or other beam. In principle, such a beam can either be decomposed directly into a VSWF representation, or into a plane wave spectrum, from which the VSWF expansion coefficients can be found using equations 
(11) and (12). In practice, this is problematic, since the usual descriptions of beams do not actually satisfy the Maxwell equations.

For the case of Gaussian beams, either plane wave expansion [7] or direct VSWF expansion [8, 9. can be used, although neither will give a beam identical to a traditional Gaussian beam.

\section{Optical forces}

Using the T-matrix method, with the T-matrix calculated by the publically available code by Mishchenko [6], and the beam shape coefficients in the localised approximation by Gouesbet 89 used to describe the beam, we calculated the variation of the axial force acting on particles of varying shape as a function of their position along the beam axis.

The particles are polystyrene $(n=1.59)$ prolate spheroids and cylinders, of varying aspect ratio as indicated (see figure 21). The particles are of equal volume, with a volume equal to that of a sphere of radius $0.75 \mu \mathrm{m}$, and are trapped in water by a Gaussian beam of waist width $0.8 \mu \mathrm{m}$ and free space wavelength $1064 \mathrm{~nm}$.

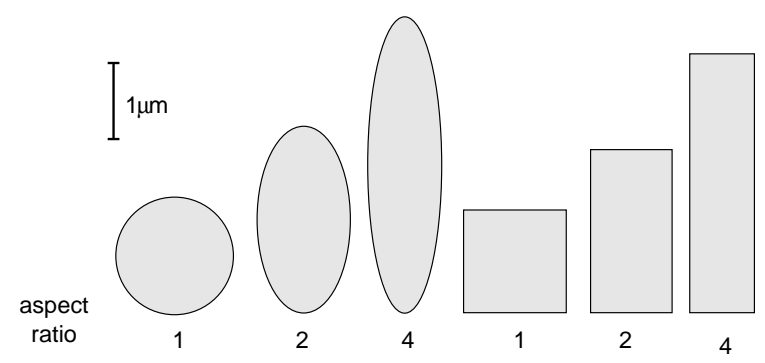

Figure 2. Differently shaped spheroidal and cylindrical particles with aspect ratios of 1,2 , and 4 .

The axial forces acting on the spheroids and cylinders are shown in figures 3 and 4 A negative position on the beam axis indicates a position before the focal plane is reached, a positive position is after the focus. A positive force acts to push the particle in the direction of propagation of the beam, a negative force will act to axially trap the particle. If only optical forces are acting, the particle will come to rest at the zero optical force position where force curve crosses the zero force line with a negative gradient.

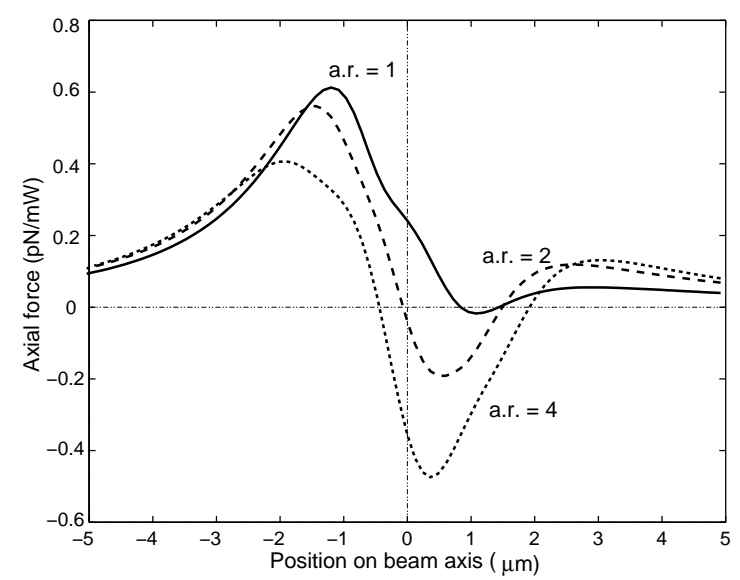

Figure 3. Axial force acting on spheroids of aspect ratios 1,2 , and 4 .

The trapping forces acting on the spheroid and cylinder with aspect ratio 1 is very small. This is due to interference due to reflections from the rear surface of the particle [11].

As the particles become smaller, the force becomes less dependent on the shape of the particle. Even for the particles considered here, it can be seen that the fine details of the shape (i.e. spheroid vs cylinder) only has a small effect on the force. As the particle becomes more elongated, it obstructs less of the trapping beam, and radiation pressure forces are reduced.

\section{Non-optical forces}

A number of non-optical forces will act on the particle. Buoyancy and gravity are constant and are simply dealt with. Motion of the particle in the surrounding fluid is completely dominated by viscous drag. 


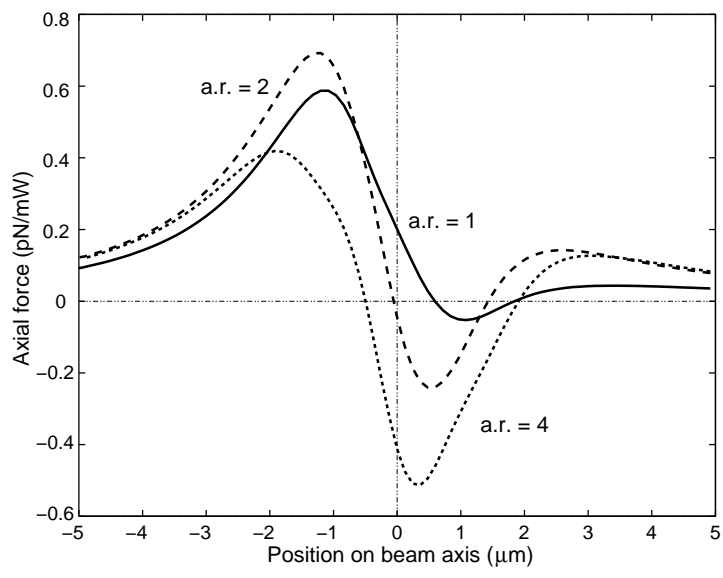

Figure 4. Axial force acting on cylinders of aspect ratios 1,2 , and 4 .

Since the time required for the particle to reach its terminal velocity of $\approx 1 \mu \mathrm{ms}^{-1}$ is very short $\left(\tau \approx 10^{-7}\right.$ s is typical $)$, we use $\dot{\mathbf{r}} \propto \mathbf{F}$ instead of $\ddot{\mathbf{r}} \propto \mathbf{F}$, since the particle will be moving at very close to the terminal velocity at all times. If the fluid is in motion, we use the velocity of the particle relative to the fluid. Since typical Reynolds numbers in trapping are extremely low ( $\mathrm{Re} \approx 10^{-5}$ is typical), perfect laminar flow can be assumed - for a spherical particle, Stoke's Law will be an excellent approximation.

In general, the trapping beam will heat the surrounding medium, possibly giving rise to convective flow. While free convection is, in general, a difficult problem, the convection problem in trapping is perhaps the simplest possible. Due to the very small distances involved, the steadystate temperature distribution is reached in a very short time [10], and a steady-state temperature distribution, independent of the convective flow, can be assumed. Similarly, any convective flow will reach steady-state very quickly.

Further effects that can be included for a complete model include Brownian motion, thermophoretic effects due to uneven heating of the particle, the effects of nearby particles and surfaces, etc. Accurate calculation of the optical forces can allow the evaluation of the accuracy of the modelling of these effects.

\section{REFERENCES}

1. B. T. Draine and P. J. Flatau, Discrete-dipole approximation for scattering calculations, J. Opt. Soc. Am. A 11 (1994) 1491-1499.

2. M. I. Mishchenko, Light scattering by randomly oriented axially symmetric particles, $\mathrm{J}$. Opt. Soc. Am. A 8 (1991) 871-882.

3. L. Tsang, J. A. Kong and R. T. Shin, Theory of microwave remote sensing, (1985) John Wiley, New York.

4. D. A. White, Vector finite element modeling of optical tweezers, Comp. Phys. Comm. 128 (2000) 558-564.

5. H. Kimura and I. Mann, Radiation pressure cross-section for fluffy aggregates, J. Quant. Spectr. Radiat. Transfer 60 (1998) 425-438.

6. M. I. Mishchenko, L. D. Travis and D. W. Mackowski, T-matrix codes for computing electromagnetic scattering by nonspherical and aggregated particles, http://www.giss.nasa.gov/〜 crmim/t_matrix.html

7. A. Doicu and T. Wriedt, Plane wave spectrum of electromagnetic beams, Opt. Comm. 136 (1997) 114-124.

8. J. A. Lock and G. Gouesbet, Rigorous justification of the localized approximation to the beam-shape coefficients in generalized Lorenz-Mie theory. I. On-axis beams, J. Opt. Soc. Am. A 9 (1994) 2503-2515.

9. G. Gouesbet and J. A. Lock, Rigorous justification of the localized approximation to the beam-shape coefficients in generalized Lorenz-Mie theory. II. Off-axis beams, J. Opt. Soc. Am. A 9 (1994) 2516-2525.

10. H. Rubinsztein-Dunlop, T. A. Nieminen, M. E. J. Friese and N. R. Heckenberg, Optical trapping of absorbing particles, Advances in Quantum Chemistry 30 (1998) 469-492.

11. P. A. Maia Neto and H. M. Nussenzveig, Theory of optical tweezers, Europhys. Lett. 50 (2000) 702-708. 